\title{
Synthesis, characterization, electrospinning and antibacterial studies on triphenylphosphine- dithiphosphonates Copper(I) and Silver(I) complexes
}

\author{
Mehmet Karakus ${ }^{1 *}$, Yuksel Ikiz ${ }^{2}$, Halil Ibrahim Kaya ${ }^{3}$ and Omer Simsek ${ }^{3}$
}

\begin{abstract}
Background: The novel amido and O-ferrocenyldithiophosphonates $\left[\mathrm{FcP}(\mathrm{S})(\mathrm{SH})\left(\mathrm{NHR} \mathrm{R}^{1}\right)\right]\left(\mathrm{FC}=\mathrm{Fe}\left(\eta^{5}-\mathrm{C}_{5} \mathrm{H}_{5}\right)\left(\eta^{5}-\mathrm{C}_{5} \mathrm{H}_{4}\right)\right.$, $\mathrm{R}^{1}=1$-(4-fluorophenylethyl and benzyloxycyclopentyl) and $\left[\mathrm{FCP}(\mathrm{S})\left(\mathrm{OR}^{2}\right) \mathrm{S}^{-}\right]\left[\mathrm{H}_{3} \mathrm{~N}^{+} \mathrm{C}\left(\mathrm{CH}_{3}\right)_{3}\right]\left(\mathrm{R}^{2}=\right.$ myrtanyl) were synthesized by the reaction of $\left[\left(F C P S_{2}\right)\right]_{2}\left(F C=F e\left(\eta^{5}-C_{5} H_{5}\right)\left(\eta^{5}-C_{5} H_{4}\right)\right)$ and chiral amines, such as (S)-(-)-1-(4fluorophenylethyl) amine and (1S,2S)-(+)-benzyloxycyclopentyl amine, and of (1S), (2S), (5S)-myrtanol in toluene. The reaction of ferrocenyldithiophosphonates and $\left[\mathrm{Cu}\left(\mathrm{PPh}_{3}\right)_{2}\right] \mathrm{NO}_{3}$ or $\mathrm{AgNO}_{3}$ and $\mathrm{PPh}_{3}$ gave rise to copper(I) and silver(l) complexes in THF. $\left[\mathrm{Ag}_{2}\left\{\mathrm{FCP}(\mathrm{OMe}) \mathrm{S}_{2}\right\}_{2}\left(\mathrm{PPh}_{3}\right)_{2}\right]$ and $\left[\mathrm{Cu}\left(\mathrm{PPh}_{3}\right)_{2}\right] \mathrm{NO}_{3}$ were embedded into nanofibers and their antimicrobial activities on fibers were also investigated.
\end{abstract}

Results: The compounds have been characterized by elemental analyses, IR, NMR ( $\left.{ }^{1} \mathrm{H}-,{ }^{31} \mathrm{P}-\right)$ spectroscopy as well as MS measurements. Nanofibers were obtained by electrospinning method which is the simplest and most effective method to produce nanoscale fibers under strong electrical field. Antimicrobial activity of the compound 5, $\mathrm{Ag}_{2}\{\mathrm{FcP}$ $\left.\left.(\mathrm{OMe}) \mathrm{S}_{2}\right\}_{2}\left(\mathrm{PPh}_{3}\right)_{2}\right]$, and $\left[\mathrm{Cu}\left(\mathrm{PPh}_{3}\right)_{2}\right] \mathrm{NO}_{3}$ on fibers were studied.

Conclusions: In this study, the new dithiophosphonate ligands were synthesized and utilized in the preparation of copper(I) and silver(I) complexes with ferrocenyldithiophosphonate and triphenylphosphine. Then, the compounds $\left[\mathrm{Ag}_{2}\left\{\mathrm{FCP}(\mathrm{OMe}) \mathrm{S}_{2}\right\}_{2}\left(\mathrm{PPh}_{3}\right)_{2}\right]$ and $\left[\mathrm{Cu}\left(\mathrm{PPh}_{3}\right)_{2}\right] \mathrm{NO}_{3}$ were added into the PAN solutions (Co-PAN dissolved in dimethylacetamide) and the solutions were electrospun onto microscope slides and PP meltblown surfaces. Antimicrobial activity of the compounds $\left[\mathrm{Ag}_{2}\left\{\mathrm{FCP}(\mathrm{OMe}) \mathrm{S}_{2}\right\}_{2}\left(\mathrm{PPh}_{3}\right)_{2}\right]$ and $\left[\mathrm{Cu}\left(\mathrm{PPh}_{3}\right)_{2}\right] \mathrm{NO}_{3}$ on fibers were determined in vitro against two indicator strains; M. luteus NCIB and E. coli ATCC25922. The obtained results indicated that these metals showed moderate level antimicrobial activities.

Keywords: Dithiophosphonates, Triphenylphosphine, Copper(I) and Silver(I) complexes, Nanofiber, Electrospinning, Antibacterial

\section{Introduction}

Metallic silver and copper are natural antimicrobial agents and historically recognized [1,2]. These agents have been added into many polymer solutions, such as polyacrilonitrile (PAN), polyvinyl alcohol (PVA), Poly(Nvinylpyrrolidone), polylactic acid (PLA), to produce nanofibers with electrospinning method [3-10].

\footnotetext{
* Correspondence: mkarakus@pau.edu.tr

${ }^{1}$ Department of Chemistry, Faculty of Arts\&Sciences, Pamukkale University, Kinikli 20075, Denizli, Turkey

Full list of author information is available at the end of the article
}

Electrospinning is a simple method to produce micro or nanoscale fibers. Nanofibers, due to their high surface area and porosity, find applications in energy storage, healthcare, biotechnology, environmental engineering, defense and especially filtration [11]. Electrospinning process uses a high voltage electric field to produce electrically charged jets from polymer solution. Polymer solution on tip of a syringe or pipette ejects toward opposite charged collector when overcome surface tension. Polymer chain entanglements prevent jets from breaking off and create fiber form. Because of evaporation 
and air drag, jets split into smaller diameters [12]. Process parameters are divided into; solution parameters which include viscosity, surface tension, electrical conductivity; processing conditions which include applied voltage, tip to collector distance, feeding amount and type; and ambient conditions which include temperature and moisture [13].

Dithiophosphonates are an important class in organophosphorus chemistry due to utilising in agricultural, medicinal and technological field [14-35]. It has been known that a considerable number of dithiophosphonates and their metal complexes have been easily synthesized by the reaction Lawesson's reagent or Ferrocenyl Lawesson's reagent and the respective alcohols or amines due to a ring opening reaction by nucleophilic attack [30-32]. However, there is no study on nanofibers of dithiophosphonates by using electrospinning method.

In the present work, we report the synthesis of novel dithiophosphonates and their metals complexes with dithiophosphonates and triphenylphosphine. All compounds were characterized by elemental analyses, IR, NMR $\left({ }^{1} \mathrm{H}-,{ }^{31} \mathrm{P}-\right)$ spectroscopy as well as MS measurements. The compounds $\left[\mathrm{Ag}_{2}\left\{\mathrm{FcP}(\mathrm{OMe}) \mathrm{S}_{2}\right\}_{2}\left(\mathrm{PPh}_{3}\right)_{2}\right]$ and $\left[\mathrm{Cu}\left(\mathrm{PPh}_{3}\right)_{2}\right] \mathrm{NO}_{3}$ added into PAN polymer solutions and mixed. Mixed polymer solutions were electrospun onto microscope slides and PP (polypropylene) meltblown surfaces. Meltblown is very commonly used textile nonwoven structure to support and protect fine fibers, especially in filtration. Antibacterial activities of those nanofibers were investigated.

\section{Result and discussion}

Synthesis and characterization

Amido and O-ferrocenyldithiophosphonates have been synthesized from Ferrocenyl Lawesson's reagent and amines or (1S,2S,5S)- (-)- O-myrtanol (Scheme 1). The Ferrocenyl Lawesson's reagent was reacted with (1S,2S,5S)(-)- O-myrtanol and a crude dithiophosphonic acid was formed and then was treated with tert-butyl amine in order to convert it to its suitable salt $\mathbf{1}$. In the case of amidodithiophoshonates 2 and 3 (Scheme 1), they were obtained as air stable solids [35]. The compound $\mathbf{5}$ was prepared by the reaction of $(R)-(+)-1$ - phenylethyl amidoferrocenyldithiophosphonate [35] and $\mathrm{AgNO}_{3}$ in toluene and $\mathrm{MeOH}$ mixture (Scheme 2).

The IR spectrum of the ligands and their complexes showed two characteristic bands at around $692-642 \mathrm{~cm}^{-1}$ and $582-515 \mathrm{~cm}^{-1}$ which are assigned to $v_{\mathrm{as}}\left(\mathrm{PS}_{2}\right)$ and $v_{\mathrm{s}}$ $\left(\mathrm{PS}_{2}\right)$, respectively $[36,37]$.

Mass spectra of the compound $\mathbf{1}-\mathbf{5}$ exhibited $\mathrm{m} / \mathrm{z}$ values for identifiable certain fragments. Specific rotations

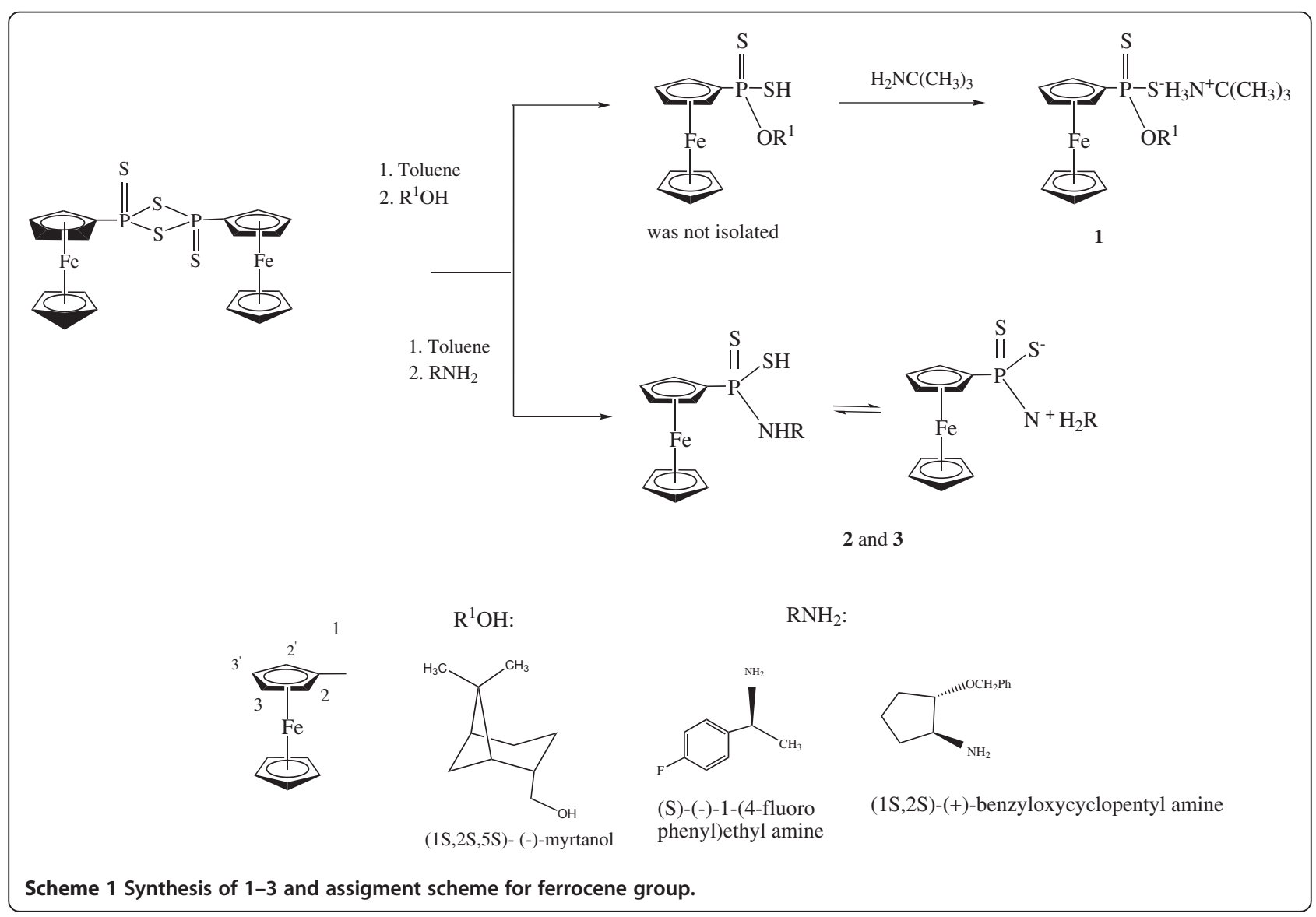




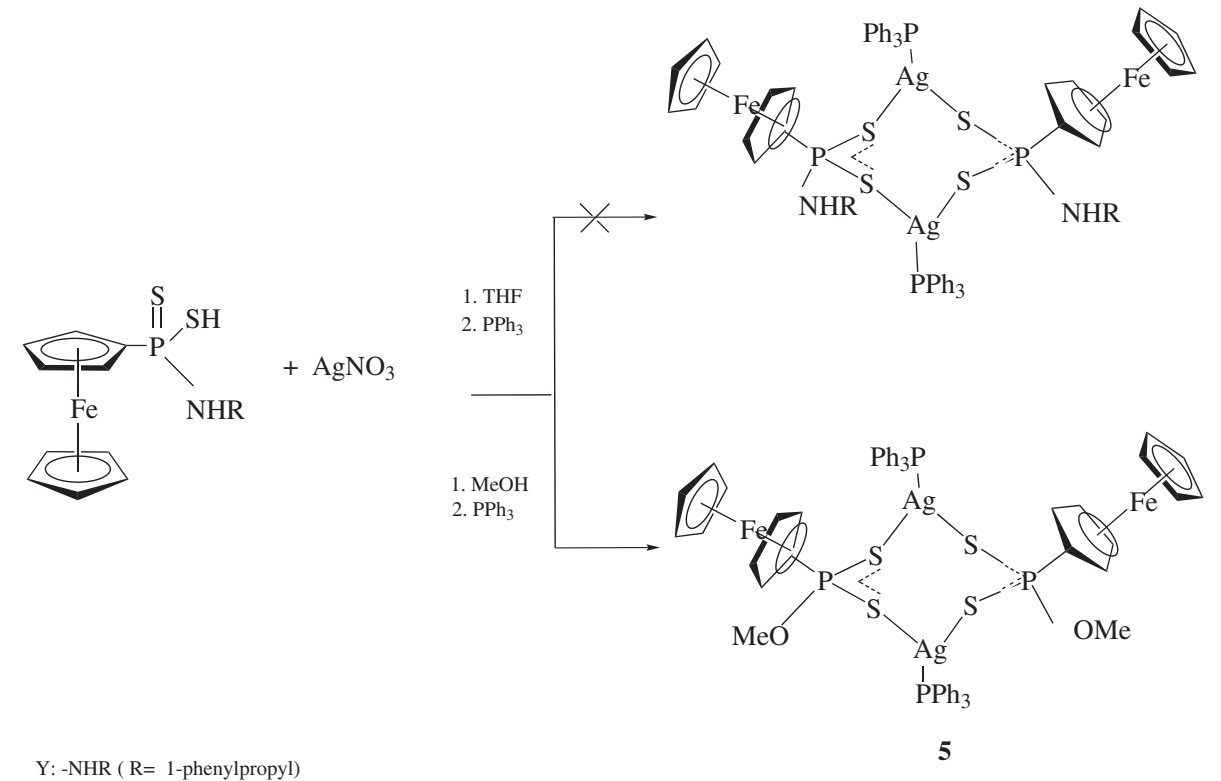

Scheme 2 Synthesis of $\left[\mathrm{Ag}_{2}\left\{\mathrm{FcP}(\mathrm{OMe}) \mathrm{S}_{2}\right\}_{2}\left(\mathrm{PPh}_{3}\right)_{2}\right] 5$.

of all compounds showed that only one optical isomer was formed.

The ${ }^{31} \mathrm{P}$ NMR spectra of the ligands $\mathbf{2}$ and $\mathbf{3}$ were measured in DMSO- $\mathrm{d}_{6}$ and showed two separate sets of signals which were shifted to $61.80 \mathrm{ppm}\left(\mathrm{J}_{\mathrm{PN}-\mathrm{H}}=41.7 \mathrm{~Hz}\right.$ for 2$)$ and $62.09 \mathrm{ppm}\left(\mathrm{J}_{\mathrm{PN}-\mathrm{H}}=38.2 \mathrm{~Hz}\right.$ for 3$)[35,38]$. A very small signal was observed in the ${ }^{31} \mathrm{P}$ NMR spectra of the ligands 2 and 3 due to probably neutral and zwitter ion form in the DMSOd6 solution (see Scheme 1 for two isomer of 2 and 3).

All ligands $\mathbf{1}-\mathbf{3}$ reported here have been characterized by elemental analysis, IR, NMR and mass spectroscopy (Additional file 1). However, the ${ }^{31} \mathrm{C}$-NMR spectra of the ligands $\mathbf{2}$ and $\mathbf{3}$ did not measured due to decomposed in the DMSO- $\mathrm{d}_{6}$.

The synthesis of copper(I) and silver(I) complexes with ferrocenyldithiophosphonate and triphenylphosphine have been described and also characterized by elemental analyses, IR, NMR and MS spectroscopies (Additional file 1). The synthesis of copper(I) complexes were performed by the reaction of $\left[\mathrm{Cu}\left(\mathrm{PPh}_{3}\right)_{2}\right] \mathrm{NO}_{3}$ and the ligands (Scheme 3).

The complex 4 was obtained as yellow-orange solid. The ${ }^{31} \mathrm{P}$ NMR spectrum of $\mathbf{4}$ showed two signals at 97.8 and $-2.9 \mathrm{ppm}$ as expected [36] which were assigned to $\mathrm{PS}_{2}$ and $\mathrm{PPh}_{3}$, respectively. The $\mathrm{Cu}(\mathrm{I})$ and $\mathrm{Ag}(\mathrm{I})$ complexes of $\mathbf{2}$ and $\mathbf{3}$ also showed two signals in the ${ }^{31} \mathrm{P}$ NMR spectrum as expected. However, other spectroscopic data were not satisfied. The ${ }^{31} \mathrm{P}$ NMR spectra of $\left[\mathrm{Ag}_{2}\left\{\mathrm{FcP}(\mathrm{OMe}) \mathrm{S}_{2}\right\}_{2}\left(\mathrm{PPh}_{3}\right)_{2}\right] \mathbf{5}$ was measured in $\mathrm{CDCl}_{3}$ and observed two signal at $92.82\left(\mathrm{PS}_{2}\right)$ and $6.03\left(\mathrm{PPh}_{3}\right) \mathrm{ppm}$.

\section{Electrospinning studies}

A comparative study on Silver(I) and Copper(I)- triphenylphosphine derivatives was performed and developed for the application of electrospun nanofibers. Figure 1 shows the compound $\left[\mathrm{Ag}_{2}\left(\mathrm{FcP}(\mathrm{OMe}) \mathrm{S}_{2}\right\}_{2}\left(\mathrm{PPh}_{3}\right)_{2}\right]$ added $\mathrm{PAN}$ nanofibers on a microscope slide and PP meltblown surface. Average fiber diameter on microscope slide was measured

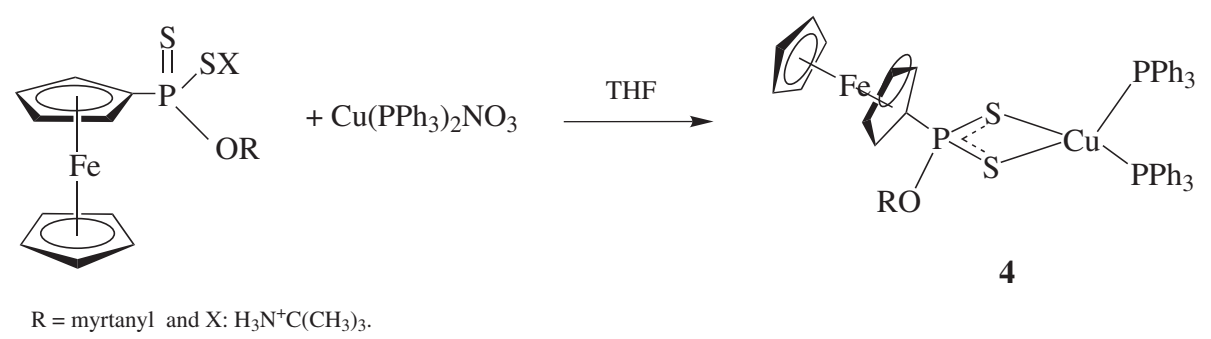

Scheme 3 Synthesis of copper(I) complex 4. 


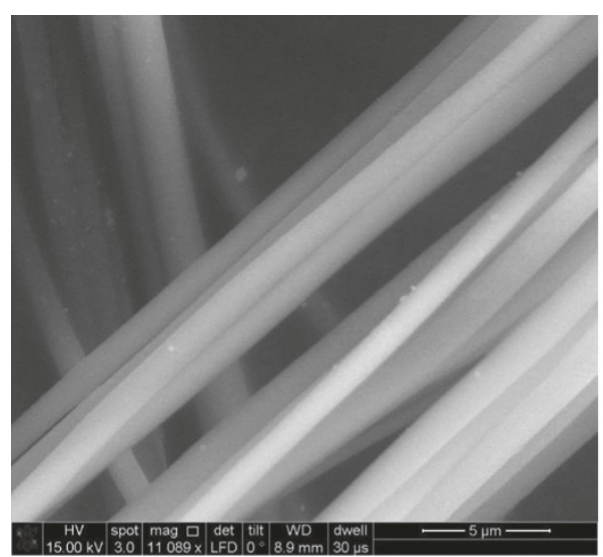

(a)

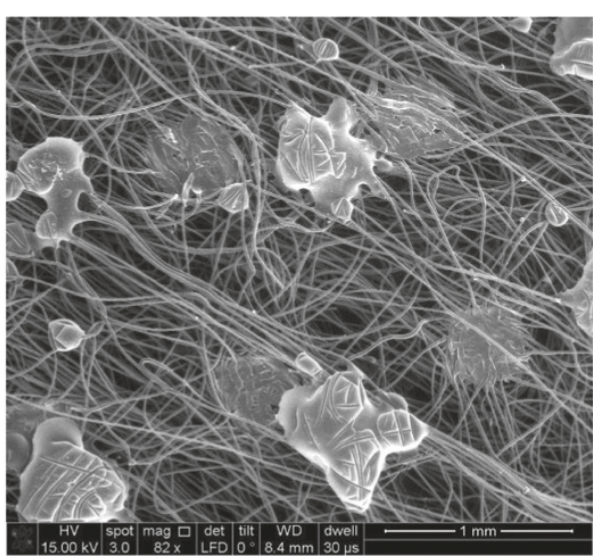

(b)

Figure $1\left[\mathrm{Ag}_{2}\left\{\mathrm{FcP}(\mathrm{OMe}) \mathrm{S}_{2}\right\}_{2}\left(\mathrm{PPh}_{3}\right)_{2}\right]$ added; a) electrospunned PAN fibers on glass, b) electrosprayed PAN particules on nonwoven surface.

about 1 micron which was higher than expected average fiber diameter. Occasional electrospraying occurred as in Figure 1-b, because of aggregation of the compound $\mathrm{Ag}_{2}$ $\left.\left\{\mathrm{FcP}(\mathrm{OMe}) \mathrm{S}_{2}\right\}_{2}\left(\mathrm{PPh}_{3}\right)_{2}\right]$ particles.

Figure 2 shows $\left[\mathrm{Cu}\left(\mathrm{PPh}_{3}\right)_{2}\right] \mathrm{NO}_{3}$ added PAN nanofibers on a microscope slide and PP meltblown surface. $\mathrm{Cu}$ particles on nanofiber surface can be seen from SEM images as in Figure 2-a. Average PP meltblown fiber diameter was measured about 15 micron.

\section{Antibacterial activities}

Antimicrobial activities of the compounds $\left[\mathrm{Ag}_{2}\{\mathrm{FcP}\right.$ $\left.\left.(\mathrm{OMe}) \mathrm{S}_{2}\right\}_{2}\left(\mathrm{PPh}_{3}\right)_{2}\right]$ and $\left[\mathrm{Cu}\left(\mathrm{PPh}_{3}\right)_{2}\right] \mathrm{NO}_{3}$ were determined first on agar media against two indicator strains; $M$. luteus NCIBM and E. coli ATCC25922. According to the well diffusion assay on agar media, $\left[\mathrm{Ag}_{2}\{\mathrm{FcP}(\mathrm{OMe})\right.$ $\left.\left.\mathrm{S}_{2}\right\}_{2}\left(\mathrm{PPh}_{3}\right)_{2}\right]$ and $\left[\mathrm{Cu}\left(\mathrm{PPh}_{3}\right)_{2}\right] \mathrm{NO}_{3}$ showed medium level of antimicrobial activities against both strains (Figure 3). When the control compounds (not including $\mathrm{Cu}$ or $\mathrm{Ag}$ derivatives) were used for the same method, no inhibition zone or no antibacterial activity was occurred meaning that the relevant antimicrobial activities were mainly due to incorporated elements of $\mathrm{Cu}$ or Ag.

The control compounds and the compounds embedded fibers on meltblown surfaces were tested for inhibition of E. coli ATCC25922 in submerged bacterial solution. The highest inhibition $(32.5 \pm 2.1 \%)$ on E. coli was achieved with the compound $\left[\mathrm{Ag}_{2}\left\{\mathrm{FCPS}_{2}(\mathrm{OMe})\right\}_{2}\right.$ $\left.\left(\mathrm{PPh}_{3}\right)_{2}\right]$. On the other hand, $\left[\mathrm{Cu}\left(\mathrm{PPh}_{3}\right)_{2}\right] \mathrm{NO}_{3}$ provided $19.4 \pm 3.2 \%$ inhibition on $E$. coli while the control compounds showed no inhibition.

In this study, the compounds showed better antibacterial activities on agar media because of diffusion. However when the compounds embedded into fibers, they showed antibacterial activities only in contact with bacteria. Even though there was limited antibacterial activity, these metals could be used on fibers with dithiphosphonate and phosphine complexes for antibacterial applications.
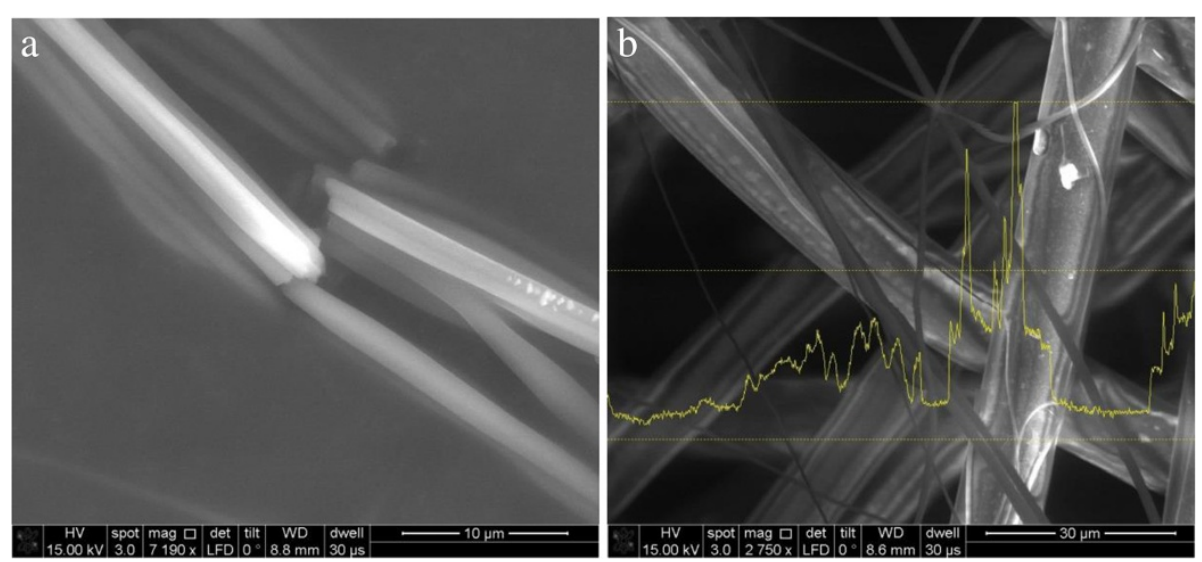

Figure $2\left[\mathrm{Cu}\left(\mathrm{PPh}_{3}\right)_{2}\right] \mathrm{NO}_{3}$ added electrospunned PAN fibers; a) on glass, b) on nonwoven surface. 


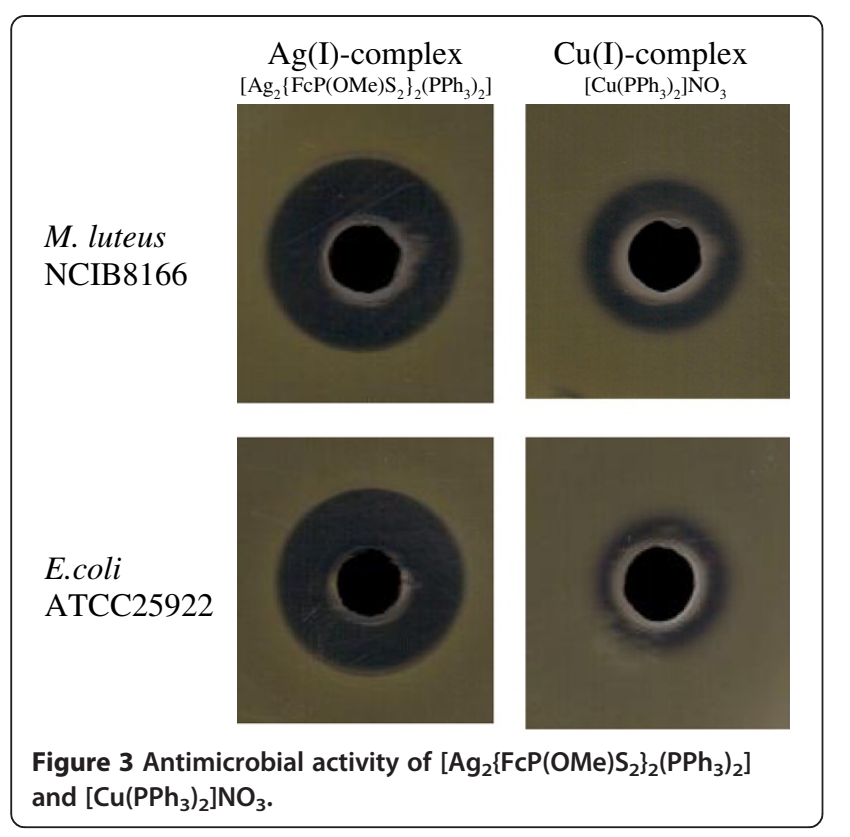

It is generally believed that heavy metals react with proteins by combining the thiol $(\mathrm{SH})$ groups, which leads to the inactivation of the proteins [39]. Therefore $\mathrm{Ag}$ and $\mathrm{Cu}$ could maintain their antimicrobial activity in the complexes of dithiphosphonate and phosphine. This is significant especially for using these metals as embedded in fibers, although they have limited antibacterial activity $[40,41]$.

\section{Experimental}

\section{Materials and method}

Solvents were distilled before used. The compounds 4 and 5 were carried out under $\mathrm{N}_{2}$ atmosphere. All other chemicals were purchased from commercial sources and used directly without further purification. $\left[\mathrm{FcPS}_{2}\right]_{2}$ (Fc: $\mathrm{Fe}\left(\eta^{5}-\mathrm{C}_{5} \mathrm{H}_{5}\right)\left(\eta^{5}-\mathrm{C}_{5} \mathrm{H}_{4}\right)$ and $\left[\mathrm{Cu}\left(\mathrm{PPh}_{3}\right)_{2}\right] \mathrm{NO}_{3}$ were prepared as described in the literature [32,42], respectively. Elemental analyses were determined with a $\mathrm{GmbH}$ varioMICRO CHNS apparatus. Melting points were determined by using Electrotermal apparatus. NMR spectra were recorded on a Bruker AVANCE DRX 400 NMR spectrometer and Jeol GSX 270 in $\mathrm{CDCl}_{3}$ and $\mathrm{d}_{6}$-DMSO. IR spectra was measured on a Perkin-Elmer 2000 FTIR spectrophotometer $\left(4000-400 \mathrm{~cm}^{-1}\right)$. Mass spectra were recorded with an AGILENT 1100 MSD and Waters machines. Optical rotation values were determined with an automatic digital ADP 440+ polarimeter.

\section{Electrospinning}

The co-polymer polyacrylonitrile (PAN) and solvent dimethylacetamide (DMAc) were obtained from "AKSA acrylic chemistry company". 15\% polymer was dissolved in $85 \%$ solvent (w/w-weight by weight basis) at 80 $100^{\circ} \mathrm{C}$ and stirred at least 4 hours. Polymer solution was prepared for electrospinning process by feeding into a pipette. Matsusada AU-40-0.75 high voltage supply were used to create electric field. Tip to collector distance was adjusted for $12 \mathrm{~cm}$ and voltage was adjusted $30 \mathrm{kV}$ between the electrodes (Figure 4).

\section{Antibacterial activity}

Two different antimicrobial test methods were used. Firstly the antimicrobial activity of synthesized compounds was determined by using well diffusion assay [43]. After filter sterilization of relevant compounds, approximately $100 \mu \mathrm{l}$ was filled to the wells which had been prepared previously by overlaying LB soft agar including the indicator strains Micrococcus luteus NCBI8166 and Escherichia coliATCC25922 on to the Müller-Hilton agar plates, then $5 \mathrm{~mm}$ wells were created with cork borer respectively. DSMO was used for controlling. To test antimicrobial efficiency of relevant compounds on fibers, the dynamic assessment of antimicrobial activity was carried out according to the standard test method released from American Society for Testing and Materials (ASTM) for immobilized antimicrobial agents under dynamic contact (E2149-01). Test bacteria (Escherichia coli ATCC25922) were cultured in LB broth (Fluka) overnight inoculations at $37^{\circ} \mathrm{C}$. Subsequently, bacterial culture was diluted in $0.3 \mathrm{mM}$ $\mathrm{KH}_{2} \mathrm{PO}_{4}$ buffer until the solution has an absorbance of $0.28 \pm 0.02$ at $475 \mathrm{~nm}$ as measured spectrophotometrically to reach bacterial suspension $\left(1.5-3.0 \times 10^{5} \mathrm{CFU} \mathrm{ml}{ }^{-1}\right)$. Rounds of fibers having total 4 in. $^{2}$ treated surface area were inoculated with $50 \pm 0.5 \mathrm{ml}$ of bacterial suspension and incubated at $37^{\circ} \mathrm{C} 1 \mathrm{~h} \pm 5 \mathrm{~min}$. Standard plate counts were performed after decimal dilution of the samples in $9 \mathrm{ml}$ of $0.1 \%$ peptone water. The percent inhibition rate

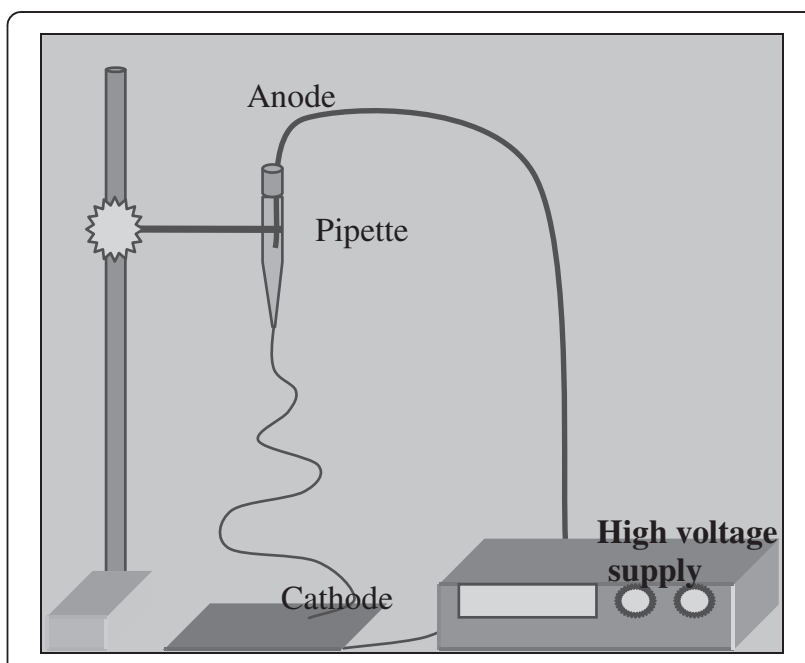

Figure 4 Electrospinning method. 
(\%) was calculated as formula of $(\mathrm{N} 1-\mathrm{N} 2 / \mathrm{N} 1) \times 100$, where $\mathrm{N} 1$ and $\mathrm{N} 2$ represent the number of colonies on the plates before and after inhibition, respectively. Untreated fibers were used as a negative control.

\section{Synthesis of ${ }^{\mathrm{t}-}$ Butyl ammonium salt of $(1 \mathrm{~S}, 2 \mathrm{~S}, 5 \mathrm{~S})-(-)-$ O-myrtanyl ferrocenyl dithiophosphonate (1)}

2,4-Bis(ferrocenyl)-1,3,2,4-dithiadiphosphetane-2,4-disulfide $[\mathrm{FcPS}(\mu-\mathrm{S})]_{2}(1.80 \mathrm{~g}, 3.21 \mathrm{mmol})$ was reacted with 1S,2S,5S)-(-)-myrtanol (1.05 g, $6.42 \mathrm{mmol}$ ) in toluene $(20 \mathrm{~mL})$. The mixture was refluxed until all solids had dissolved. The dark brown solution was cooled to rt, filtered and treated with excess tert-butyl amine. The product was precipitated in freezer from toluene as a yellow solid, which was isolated by filtration, washed with toluene and $n$-hexane and then dried in air. Yield: 2.10 g $65 \%$, m.p.: $>187(\text { dec. })^{\circ} \mathrm{C}$. $[\boldsymbol{\alpha}]_{589}^{25}=-3.61$ (c $=0.55$ in THF). IR(KBr, cm $\left.{ }^{-1}\right) v_{\max }: 648\left(\mathrm{~s}, \mathrm{PS}_{2}\right.$, asym) and 582 (m, $\mathrm{PS}_{2}$, sym). ${ }^{1} \mathrm{H}$ NMR (DMSO-d $\left.\mathrm{d}_{6}, \mathrm{ppm}\right) \delta: 4.42$ (br, $\left.2 \mathrm{H}, \mathrm{C}_{5} \mathrm{H}_{4}\right), 4.23\left(\mathrm{~s}, 5 \mathrm{H}, \mathrm{C}_{5} \mathrm{H}_{5}\right), 4.21$ (br, $\left.2 \mathrm{H}, \mathrm{C}_{5} \mathrm{H}_{5}\right), 4.18$ (br, $2 \mathrm{H}, \mathrm{OCH}_{2}$ ), 1.80-1.25 (m, 9H in myrtanyl group), $1.18(\mathrm{~s}, 9 \mathrm{H}, t \mathrm{Bu}), 1.02\left(\mathrm{~s}, 3 \mathrm{H}, \mathrm{CH}_{3}\right), 1.01\left(\mathrm{~s}, 3 \mathrm{H}, \mathrm{CH}_{3}\right) \cdot{ }^{13} \mathrm{C}$ NMR (DMSO- $\left.\mathrm{d}_{6}, \mathrm{ppm}\right) \delta$ : $90.94\left(\mathrm{~d}, \mathrm{C}^{1}\right.$; ipso-C in $\mathrm{C}_{5} \mathrm{H}_{4}$, $\left.{ }^{1} J_{\mathrm{P}, \mathrm{C}}=124.7 \mathrm{~Hz}\right), 84.23\left(\mathrm{~d},{ }^{2} \mathrm{~J}_{\mathrm{P}, \mathrm{C}}=7.9 \mathrm{~Hz}\right), 71.30\left(\mathrm{~d}, \mathrm{C}^{2}\right.$ and $\left.\mathrm{C}^{2},{ }^{2} J_{\mathrm{P}, \mathrm{C}}=13.9 \mathrm{~Hz}\right), 70.06\left(\mathrm{~s}, \mathrm{C}_{5} \mathrm{H}_{5}\right), 69.71\left(\mathrm{~d},{ }^{4} J_{\mathrm{P}, \mathrm{C}}=\right.$ $2.7 \mathrm{~Hz}), 68.91\left(\mathrm{~d}, \mathrm{C}^{3}\right.$ and $\left.\mathrm{C}^{3},{ }^{3} \mathrm{~J}_{\mathrm{P}, \mathrm{C}}=4.9 \mathrm{~Hz}\right), 49.81(\mathrm{~s}$, $t$ But), $49.12\left(\mathrm{~d},{ }^{3} J_{\mathrm{P}, \mathrm{C}}=5.2 \mathrm{~Hz}\right), 48.37,41.50,29.95(\mathrm{~s}, t \mathrm{Bu})$, 29.71, 26.78, 26.41, 22.23, 20.8 ppm. ${ }^{31}$ P NMR (DMSO$\left.\mathrm{d}_{6} \mathrm{ppm}\right) \delta: 105.46 . \mathrm{MS}(\mathrm{ESI}): \mathrm{m} / \mathrm{z} 433.1\left[\mathrm{M}-\left(\mathrm{H}_{3} \mathrm{~N}^{+} \mathrm{C}\right.\right.$ $\left.\left(\mathrm{CH}_{3}\right)_{3}\right]$. Anal. Calcd. for $\mathrm{C}_{24} \mathrm{H}_{38} \mathrm{FeNOPS}_{2}: \mathrm{C}, 56.80 ; \mathrm{H}$, 7.55; N, 2.76; S, 12.64\%. Found: C, 57.08; H, 7.38; N, 2.72; S, $12.18 \%$.

\section{Synthesis of (S) -(-)-1-(4-fluorophenylethyl)- amidoferrocenyldithiophoshonate (2)}

$[\mathrm{FcP}(\mathrm{S})(\mu-\mathrm{S})]_{2} 1.50 \mathrm{~g}(2.67 \mathrm{mmol})$ was treated with $(\mathrm{S})-$ (-)-1-(4-fluorophenylethyl) amine (0.745 g, $5.35 \mathrm{mmol})$ in a 1:2 ratio in toluene $(25 \mathrm{~mL})$ to give the corresponding amidoferrocenyldithiophosphonate. The reaction was carefully heated until all the solids dissolved and a brown solution was obtained and then a solid product was formed, which was isolated by filtration. The product was washed with petroleum ether $\left(40-60^{\circ} \mathrm{C}\right)$. The yellow crystalline product was dried under vacuum. Yield: 1.57 g, $70 \%$, m.p.: $169^{\circ} \mathrm{C}$. $[\alpha]_{589}^{25}=75$ (c $=0.08$ in THF). IR(KBr, $\left.\mathrm{cm}^{-1}\right) v_{\max }: 645\left(\mathrm{~s}, \mathrm{PS}_{2}\right.$, asym) and 526 (m, $\mathrm{PS}_{2}$, sym). ${ }^{1} \mathrm{H}$ NMR (DMSO-d $\left.{ }_{6} \mathrm{ppm}\right) \delta: 7.63$ (br, $2 \mathrm{H}$, arom.), 7.25 (br, $2 \mathrm{H}$, arom.), 4.56 (br, $2 \mathrm{H}, \mathrm{C}_{5} \mathrm{H}_{4}$ ), 4.43 (br, $\left.2 \mathrm{H}, \mathrm{C}_{5} \mathrm{H}_{4}\right), 4.37\left(\mathrm{~s}, 5 \mathrm{H}, \mathrm{C}_{5} \mathrm{H}_{5}\right), 2.50\left(\mathrm{~s}, 3 \mathrm{H}, \mathrm{CH}_{3}\right), 1.59$ (s, $1 \mathrm{H}, \mathrm{CH}) .{ }^{31} \mathrm{P}$ NMR $\left(\mathrm{DMSO}-\mathrm{d}_{6} \mathrm{ppm}\right) \delta: 61.80\left(\mathrm{~d}, \mathrm{~J}_{\mathrm{PNH}}=\right.$ $41.7 \mathrm{~Hz}) \mathrm{ppm}$. MS (ESI): $\mathrm{m} / \mathrm{z}=401.95[\mathrm{M}-\mathrm{F}]^{+}$. Anal. Calcd. for $\mathrm{C}_{18} \mathrm{H}_{19} \mathrm{NFPS}_{2} \mathrm{Fe}$ : C, 51.56; H, 4.57; N, 3.34; S, 15.29\%. Found: C, 51.71; H, 5.07; N, 3.54; S, 14.20\%.
Synthesis of $(1 \mathrm{~S}, 2 \mathrm{~S})-(+)-$-benzyloxycyclopentylamidoferrocenyldithiophoshonate (3)

Compound 3 was prepared in the same manner as compound 2, from $[\mathrm{FcP}(\mathrm{S})(\mu-\mathrm{S})]_{2}(1.00 \mathrm{~g}, 1.78 \mathrm{mmol})$ and 1S,2S-(+)-benzyloxycyclopentyl amine $0.68 \mathrm{~g}(3.56 \mathrm{mmol})$ in toluene $(25 \mathrm{~mL})$. Yield: $1.19 \mathrm{~g}(76 \%)$, m.p.: $174-176^{\circ} \mathrm{C}$. $[\boldsymbol{\alpha}]_{589}^{25}=53.33$ (c $=0.15$ in THF). IR $\left(\mathrm{KBr}, \mathrm{cm}^{-1}\right) v_{\max }$ : $645\left(\mathrm{~s}, \mathrm{PS}_{2}\right.$, asym) and $525\left(\mathrm{~m}, \mathrm{PS}_{2}\right.$, sym). ${ }^{1} \mathrm{H}$ NMR (DMSO- $\left.\mathrm{d}_{6} \mathrm{ppm}\right) \delta: 8.29(\mathrm{br}, 1 \mathrm{H}, \mathrm{NH}), 7.37(\mathrm{br}, 5 \mathrm{H}$, arom.), 4.54 (br, s, $\left.2 \mathrm{H}, \mathrm{C}_{5} \mathrm{H}_{4}\right), 4.21$ (br, s, $5 \mathrm{H}, \mathrm{C}_{5} \mathrm{H}_{5}$ ), 4.18 (br, s, $2 \mathrm{H}, \mathrm{C}_{5} \mathrm{H}_{4}$ ), 3.99 (br, $2 \mathrm{H}, \mathrm{OCH}_{2}$ ), $3.80-1.69$ (br, $\mathrm{m}, 8 \mathrm{H}, \mathrm{C}_{5} \mathrm{H}_{8}$ group). ${ }^{31} \mathrm{P}$ NMR (DMSO-d $\left.\mathrm{d}_{6} \mathrm{ppm}\right) \delta$ : $62.09 \mathrm{ppm}\left(\mathrm{J}_{\mathrm{PN}-\mathrm{H}}=38.2 \mathrm{~Hz}\right) \mathrm{ppm}$. MS (ESI): $\mathrm{m} / \mathrm{z}=$ $296.86\left[\mathrm{M}-\mathrm{C}_{5} \mathrm{H}_{8} \mathrm{OCH}_{2} \mathrm{C}_{6} \mathrm{H}_{5}\right]^{+}$. Anal. Calcd. for $\mathrm{C}_{22} \mathrm{H}_{27}$ $\mathrm{NOPS}_{2} \mathrm{Fe}$ : C, 56.06; H, 5.59; N, 2.97\%. Found: C, 60.07; $\mathrm{H}, 6.34 ; \mathrm{N}, 3.30 \%$.

\section{Synthesis of $\left[\mathrm{Cu}\left\{\mathrm{Fe}\left(\eta^{5}-\mathrm{C}_{5} \mathrm{H}_{5}\right)\left(\eta^{5}-\mathrm{C}_{5} \mathrm{H}_{4} \mathrm{P}(\mathrm{OR}) \mathrm{S}_{2}\right)\left(\mathrm{PPh}_{3}\right)_{2}\right\}\right]$ $(\mathrm{R}=$ myrtanyl) (4)}

A solution of $\left[\mathrm{Cu}\left(\mathrm{PPh}_{3}\right)_{2} \mathrm{NO}_{3}\right](0.13 \mathrm{~g}, 0.20 \mathrm{mmol})$ in THF $(10 \mathrm{~mL})$ was added dropwise to a solution of $(1 \mathrm{~S}, 2 \mathrm{~S}, 5 \mathrm{~S})-$ O-myrtanyl-ferrocenyldithiophoshonate $1(0.10$ g, 0.20 $\mathrm{mmol})$ in THF $(10 \mathrm{~mL})$ and stirred at r.t. for $2 \mathrm{~h}$. A yelloworange solution was observed. The reaction mixture was filtered and the solvent was removed under reduced pressure. A yellow-orange crystalline product was isolated. Yield: 0.12 g, $60 \%$, m.p.: $179-180^{\circ} \mathrm{C}$. $[\boldsymbol{\alpha}]_{589}^{25}=120$ (c = 0.05 in THF). IR (KBr, cm $\left.{ }^{-1}\right) v_{\max }: 642\left(\mathrm{~s}, \mathrm{PS}_{2}\right.$, asym) and 515 (m, $\left.\mathrm{PS}_{2}, \mathrm{sym}\right) .{ }^{1} \mathrm{H}$ NMR $\left(\mathrm{CDCl}_{3}, \mathrm{ppm}\right) \delta: 7.43-7.25$ (m, $30 \mathrm{H}$, arom.), 4.36 (br, $\left.2 \mathrm{H}, \mathrm{C}_{5} \mathrm{H}_{4}\right), 4.25$ (s, $\left.2 \mathrm{H}, \mathrm{C}_{5} \mathrm{H}_{5}\right), 4.21$ (s, $\left.2 \mathrm{H}, \mathrm{C}_{5} \mathrm{H}_{4}\right), 3.80(\mathrm{~m}, 2 \mathrm{H}, \mathrm{OCH} 2), 2.40-1.10(\mathrm{~m}, 9 \mathrm{H}$, in myrtanyl group), $1.24\left(\mathrm{~s}, 3 \mathrm{H}, \mathrm{CH}_{3}\right), 0.87\left(\mathrm{~s}, 3 \mathrm{H},-\mathrm{CH}_{3}\right) .{ }^{31} \mathrm{P}$ $\mathrm{NMR}\left(\mathrm{CDCl}_{3}, \mathrm{ppm}\right) \delta: 97.85\left(\mathrm{PS}_{2}\right)$ and $-2.87\left(\mathrm{PPh}_{3}\right) \mathrm{ppm}$. Anal. Calcd. for $\mathrm{C}_{56} \mathrm{H}_{56} \mathrm{OP}_{3} \mathrm{~S}_{2} \mathrm{FeCu}\left(1021.51 \mathrm{~g} \cdot \mathrm{mol}^{-1}\right)$ : C, 65.84; H, 5.52; S, 6.27\%. Found: C, 65.49; H, 5.54; S, 5.93\%.

\section{Synthesis of $\left[\mathrm{Ag}\left\{\mathrm{Fe}\left(\eta^{5}-\mathrm{C}_{5} \mathrm{H}_{5}\right)\left(\eta^{5}-\mathrm{C}_{5} \mathrm{H}_{4} \mathrm{P}(\mathrm{OR}) \mathrm{S}_{2}\right)\left(\mathrm{PPh}_{3}\right)_{2}\right\}\right]_{2}$ $\left(\mathrm{R}=\mathrm{CH}_{3}\right)(5)$}

A mixture of $\mathrm{AgNO}_{3}(0.12 \mathrm{~g}, 0.70 \mathrm{mmol})$ and $\mathrm{PPh}_{3}$ $(0.18 \mathrm{~g}, 0.70 \mathrm{mmol})$ in $\mathrm{MeOH}(20 \mathrm{~mL})$ was added dropwise to a solution of the compound (R) - $(+)-1$ - Phenylethyl amidoferrocenyldithiophosphonate [35] (0.28 g, $0.70 \mathrm{mmol})$ in toluene $(25 \mathrm{~mL})$ and stirred for $2 \mathrm{~h}$. A yellow precipitate product was immediately formed. The product was filtered, washed with petroleum ether $(40-$ $\left.60^{\circ} \mathrm{C}\right)$ and dried in air. Yield: $0.38 \mathrm{~g}(79 \%)$. M.p.: $>160^{\circ} \mathrm{C}$ (dec.). $\operatorname{IR}\left(\mathrm{KBr}, \mathrm{cm}^{-1}\right) v_{\text {max }}: 649\left(v_{\text {asym }} \mathrm{PS}_{2}\right)$ and $560\left(v_{\text {sym }}\right.$ $\left.\mathrm{PS}_{2}\right) .{ }^{1} \mathrm{H} \mathrm{NMR}\left(\mathrm{CDCl}_{3}, \mathrm{ppm}\right) \delta: 7.36-7.02(\mathrm{~m}, 30 \mathrm{H}$, arom.), 4.55 (br, $\left.4 \mathrm{H}, 2 \times \mathrm{C}_{5} \mathrm{H}_{4}\right), 4.36$ (br, $4 \mathrm{H}, 2 \mathrm{xC}_{5} \mathrm{H}_{4}$ ), $4.16\left(\mathrm{~s}, \mathrm{br}, 10 \mathrm{H}, 2 \mathrm{xC}_{5} \mathrm{H}_{5}\right), 1.39\left(\mathrm{~d}, \mathrm{br}, 6 \mathrm{H}, 2 \mathrm{xOCH}_{3},{ }^{3} \mathrm{~J}_{\mathrm{P}, \mathrm{H}}=\right.$ 5.4 Hz). ${ }^{31} \mathrm{P}$ NMR $(\mathrm{CDCl}, \mathrm{ppm}) \delta: 97.82\left(\mathrm{PS}_{2}\right)$ and 6.03 $\left(\mathrm{PPh}_{3}\right)$. MS (ESI) $(\mathrm{m} / \mathrm{z}): 279.1\left[\mathrm{FcPS}_{2}\right]^{+}$. Anal. Calc. for $\mathrm{C}_{58} \mathrm{H}_{54} \mathrm{O}_{2} \mathrm{P}_{4} \mathrm{~S}_{4} \mathrm{Fe}_{2} \mathrm{Ag}_{2}$ : C, 51.12; H, 3.99; S, 9.41. Found: $\mathrm{C}$, 50.76; H, 3.96; S, 9.87\%. 


\section{Conclusions}

The new dithiophosphonate ligands were synthesized and utilized in the preparation of copper(I) and silver(I) complexes with ferrocenyldithiophosphonate and triphenylphosphine. Then, the compounds $\left[\mathrm{Ag}_{2}\left\{\mathrm{FcP}(\mathrm{OMe}) \mathrm{S}_{2}\right\}_{2}\right.$ $\left.\left.\mathrm{PPh}_{3}\right)_{2}\right]$ and $\left[\mathrm{Cu}\left(\mathrm{PPh}_{3}\right)_{2} \mathrm{NO}_{3}\right]$ were added into the PAN polymer solution (Co-PAN dissolved in dimethylacetamide) and the solution was electrospun onto microscope slide and PP meltblown surface producing fibers, average about 1 micron diameter. SEM images of these fibers show that compounds did not evenly distribute on fiber surface along the fiber length, meaning also not evenly distributed in polymer solution because of particles aggregation which caused electrospraying, as well. Antimicrobial activity of the compounds $\left(\left[\mathrm{Ag}_{2}\left\{\mathrm{FcPS}_{2}(\mathrm{OMe})\right\}_{2}\right.\right.$ $\left.\left(\mathrm{PPh}_{3}\right)_{2}\right]$ and $\left.\left[\mathrm{Cu}\left(\mathrm{PPh}_{3}\right)_{2}\right] \mathrm{NO}_{3}\right)$ on fibers were determined in vitro against two indicator strains; $M$. luteus NCIB and E. coli ATCC25922. The obtained results indicated that these metals could be immobilized with the dithiophosphonate-phophine and showed moderate level antimicrobial activity.

\section{Additional file}

\section{Additional file 1: Spectra of Compounds.}

\section{Competing interests}

The authors declare that they have no competing interests.

\section{Authors' contributions}

MK has coordinated the experimental work, synthesized, characterized the structure of the all compounds and wrote the manuscript. YI has obtained nanofiber by electrospinning method. HIK and OS carried out antibacterial studies. All authors have read and approved the final manuscript.

\section{Acknowledgment}

This study was supported by Turkish Council of Research and Technology, TUBITAK (Grant no: 107 T817).

\section{Author details}

'Department of Chemistry, Faculty of Arts\&Sciences, Pamukkale University, Kinikli 20075, Denizli, Turkey. ${ }^{2}$ Department of Textile Engineering, Faculty of Engineering, Pamukkale University, Kinikli 20075, Denizli, Turkey. ${ }^{3}$ Department of Food Engineering, Faculty of Engineering, Pamukkale University, Kinikli 20075, Denizli, Turkey.

Received: 17 December 2013 Accepted: 5 March 2014 Published: 14 March 2014

\section{References}

1. Silvestry-Rodrigues N, Sicairos-Ruelas EE, Gerba EE, Bright KR: Silver as a disinfectant. Rev Environ Contam Toxicol 2007, 191:23-45.

2. Grass G, Rensing C, Solioz M: Metallic copper as an antimicrobial surface. Appl Environ Microb 2011, 77:1541-1547.

3. Yang QB, Li DM, Hong YL, Li ZY, Wang C, Qiu SL, Wei Y: Preparation and characterization of a pan nanofibre containing Ag nanoparticles via electrospinning. Synthetic Met 2003, 137:973-974.

4. Lee HK, Jeong EH, Baek CK, Youk JH: One-step preparation of ultrafine poly(acrylonitrile) fibers containing silver nanoparticles. Mater Lett 2005, 59:2977-2980.

5. Jin WJ, Jeon HJ, Kim JH, Youk JH: A study on the preparation of poly(vinyl alcohol) nanofibers containing silver nanoparticles. Synthetic Met 2007, 157:454-459.
6. Hong $\mathrm{KH}$ : Preparation and properties of electrospun poly (vinyl alcohol)/ silver fiber web as wound dressings. Polym Eng Sci 2007, 47:43-49.

7. Jin WJ, Lee HK, Jeong EH, Park WH, Youk JH: Preparation of polymer nanofibers containing silver nanoparticles by using poly ( $\mathrm{N}$ vinylpyrrolidone). Macromol Rapid Comm 2005, 26:1903-1907.

8. Yousef A, Barakat NAM, Amna T, Al-Deyab SS, Hassan MS, Abdel-hay A, Kim HY: Inactivation of pathogenic Klebsiella pneumoniae by $\mathrm{CuO} / \mathrm{TiO} 2$ nanofibers: A multifunctional nanomaterial via one-step electrospinning. Ceram Int 2012, 38:4525-4532.

9. Xu X, Yang $Q$, Wang $Y, Y u$ H, Chen $X$, Jing X: Biodegradable electrospun poly(L-lactide) fibers containing antibacterial silver nanoparticles. Eur Polym J 2006, 42:2081-2087.

10. Han XJ, Huang ZM, Huang C, Du ZF, Wang H, Wang J, He CL, Wu QS: Preparation and characterization of electrospun polyurethane/inorganicparticles nanofibers. Polym Composites 2045, 2012:33.

11. Ramakrishna S, Fujihara K, Teo WE, Yong T, Ma Z, Ramaseshan R: Electrospun nanofibers solving global issues. Mater Today 2006, 9:40-57.

12. Reneker $\mathrm{DH}$, Chun I: Nanometre diameter fibres of polymer, produced by electrospinning. Nanotechnology 1996, 7:216-223.

13. Ramakrishna S: Introduction to Electrospinning and Nanofibers. Singapore: World Scientific Publishing; 2005.

14. Contarini S, Tripaldi G, Ponti G, Lizzit S, Baraldi A, Paolucci G: Surface investigation of lubricant-metal interactions by synchrotron photoemission spectroscopy. App/ Surf Sci 1997, 108:359-364.

15. Fuller M, Yin Z, Kasrai M, Bancroft GM, Yamaguchi ES, Ryason PR, Willermet $\mathrm{PA}$, Tan $\mathrm{KH}$ : Chemical characterization of tribochemical and thermal films generated from neutral and basic ZDDPs using X-ray absorption spectroscopy. Tribol Int 1997, 30:305-315.

16. Ma Q, Wang X-Y, Chen $Q$, Leung W-H, Zhang Q-F: Dinuclear ruthenium complexes containing tripodal dithiophosphonate ligands. Inorg Chimica Acta 2011, 378:148-153.

17. Karakus M, Loennecke P, Hildebrand M, Hey-Hawkins E: Chiral Heterobimetallic Gold(I) Ferrocenyldithiophosphonato Complexes. Z Anorg Allg Chem 2011, 637:983-987.

18. Karakus M, Lönnecke $P$, Hey-Hawkins E: Zwitterionic ferrocenyldithiophosphonates: the molecular structure of $\left[\mathrm{FcP}(\mathrm{S}) \mathrm{S}\left(\mathrm{OCH}_{2} \mathrm{CH}_{2} \mathrm{NH}_{2} \mathrm{Me}\right)\right][\mathrm{Fc}=\mathrm{Fe}$ $\left.\left(\eta^{5}-\mathrm{C}_{5} \mathrm{H}_{4}\right)\left(\eta^{5}-\mathrm{C}_{5} \mathrm{H}_{5}\right)\right]$. Polyhedron 2004, 23:2281-2284.

19. Karakus M: Synthesis and characterization of novel organothiophosphorus compounds: X-ray crystal structure of $\mathrm{H}_{3} \mathrm{COC}_{6} \mathrm{H}_{4} \mathrm{P}\left(\mathrm{OC}_{2} \mathrm{H}_{4} \mathrm{~S}\right)(\mathrm{S})$ synthesized by a new method. $Z$ Anorg Allg Chem 2006, 632(8-9):1549-1553.

20. Karakus M, Yilmaz H, Bulak E, Lönnecke P: Bis\{u-[O-cyclopenthyl(4methoxyphenyl)dithiophosphonato]1к:S 2K S -[O-cyclopenthyl(4methoxyphenyl)dithiophosphonato]-1K2S, S'\}dizinc(II). Appl Organomet Chem 2005, 19:396-397.

21. van Zyl WE, Woollins JD: The coordination chemistry of dithiophosphonates: An emerging and versatile ligand class. Coord Chem Rev 2013, 257:718-731

22. van Zyl WE: Dithiophosphonates and related P/S-type ligands of group 11 metals. Comments Inorg Chem 2010, 31:13-45.

23. van Zyl WE, Fackler JP Jr: A general and convenient route to dithiophosphonate salt derivatives. Phosphorus, Sulfur, Silicon Relat Elem 2000, 167:117-132.

24. van Zyl WE, López-de-Luzuriaga JM, Fackler JP Jr: Luminescence studies of dinuclear gold(I) phosphor-1,1-dithiolate complexes. J Mol Struct 2000, 516:99-106

25. van ZyI WE, Staples RJ, Fackler JP Jr: Dinuclear gold(I) dithiophosphonate complexes: formation, structure, and reactivity. Inorg Chem Commun 1998, 1:51-54

26. Pillay MN, Omondi B, Staples RJ, van Zyl WE: A hexanuclear gold(I) metallatriangle derived from a chiral dithiophosphate: synthesis, structure, luminescence and oxidative bromination reactivity. CrystEngComm 2013, 15:4417-4421.

27. Pollnitz A, Silvestru A, Gimeno MC, Laguna A: New gold(I) and silver(I) complexes with organophosphorus ligands with SPNSO skeleton. Crystal and molecular structures of monomeric $\left[\mathrm{Au}\left\{\left(\mathrm{SPPh}_{2}\right)\left(\mathrm{O}_{2} \mathrm{SR}\right) \mathrm{N}\right\}\left(\mathrm{PPh}_{3}\right)\right](\mathrm{R}=$ $\left.\mathrm{Me}, \mathrm{C}_{6} \mathrm{H}_{4} \mathrm{Me}-4\right)$ and dimeric $\left.\left[\mathrm{Ag}\left\{\left(\mathrm{SPPh}_{2}\right)\left(\mathrm{O}_{2} \mathrm{SPh}\right) \mathrm{N}\right\}\left(\mathrm{PPh}_{3}\right)\right]_{2} \cdot 2 \mathrm{CH}_{2} \mathrm{Cl}_{2}\right]$. Inorg Chimica Acta 2010, 363:346-352.

28. Liu S-L, Wang X-Y, Duan T, Leung W-H, Zhang Q-F: Hydrolysis and coordination behavior of ferrocenyl-phosphonodithiolate: Synthesis and structure of $\mathrm{Cu}_{4}\left[\mathrm{FCP}\left(\mathrm{OCH}_{3}\right)(\mu-\mathrm{S})\left(\mu_{3}-\mathrm{S}\right)\right]_{4}\left[\mathrm{FC}=\mathrm{Fe}\left(\eta^{5}-\mathrm{C}_{5} \mathrm{H}_{4}\right)\left(\eta^{5}-\mathrm{C}_{5} \mathrm{H}_{5}\right)\right]$. J Mol Struct 2010, 964:78-81 
29. Barranco EM, Crespo O, Gimeno MC, Jones PG, Laguna A: Unprecedented formation of novel phosphonodithioate ligands from diferrocenyldithiadiphosphetane disulfide. Inorg Chem 2008, 47:6913-6918.

30. Gray IP, Milton HL, Slawin AMZ, Woollins JD: Synthesis and structure of [Fc(RO)PS $]^{-}$complexes. Dalton Trans 2003, 17:3450-3457.

31. Gray IP, Milton HL, Slawin AMZ, Woollins JD: Synthesis and structure of [An(RO)PS $\left.]_{2}\right]^{-}$complexes. Dalton Trans 2004, 16:2477-2486.

32. Foreman MRSJ, Slawin AMZ, Woollins JD: The reaction of dithiadiphosphetane disulfides with dienes, alkenes and thioaldehydes. J Chem Soc Dalton Trans 1999, 7:1175-1184.

33. Solak S, Aydemir C, Karakus M, Lönnecke P: Novel Gold(I) and Silver(I) Complexes of Phosphorus-1,1,-dithiolates and Molecular Structure of [O, O'-(Bornyl)2PS2]H3NC(CH3). Chem Cen J 2013, 7:89.

34. Herna'ndez-Galindo M del C, Jancik V, Moya-Cabrera MM, Toscano RA, Cea-Olivares $\mathrm{R}$ : 2D hydrogen bond networks in the crystals of $\left[\left(\mathrm{NH}_{4}\right.\right.$ center dot $\mathrm{H} 2 \mathrm{O})(2)][(\mathrm{RO})(\mathrm{Fc}) \mathrm{P}(\mathrm{S})(2)](2)(\mathrm{R}=3-(\mathrm{BzO})-\mathrm{Bz}, 4-(\mathrm{n}-\mathrm{Bu})-\mathrm{Bz}, \mathrm{Bz}=$ benzyl). J Organomet Chem 2007, 692:5295-5304.

35. Karakus M: Synthesis and Characterization of Chiral Gold(I) Phosphine Complexes with New Dithiophosphorus Ligands. Phosphorus, Sulfur, Silicon Relat Elem 2011, 186:1523-1530.

36. Haiduc I, Mezei G, Micu - Semeniuc R, Edelman FT, Fisher A: Differing coordination modes of (O-alkyl)-p-ethoxyphenyldithiophosphonato ligands in copper(I), silver(I) and gold(I) triphenylphosphine complexes. Z Anorg Allg Chem 2006, 632:295-300.

37. Haiduc I, Sowerby DB, Lu S-F: Stereochemical aspects of phosphor-1,1dithiolato metal complexes (dithiophosphates, dithiophosphinates): coordination patterns, molecular structures and supramolecular associations - I. Polyhedron 1995, 14:3389-3472.

38. Cui Z, Miao Z, Zhang J, Chen R-Y: Synthesis of Diphenyl alpha-(O-phenyl bis(2-chloroethyl) amidophosphorylamino)- phosphonates. Phosphorus, Sulfur, Silicon Relat Elem 2008, 183:720-725.

39. Silver S, Phung LT: Bacterial heavy metal resistance: new surprises Annu Rev Microbiol. Annu Rev Microbiol 1996, 50:753-789.

40. Windler $L$, Height $M$, Nowack B: Comparative evaluation of antimicrobials for textile applications. Environ Int 2013, 53:62-73.

41. McArthur JV, Tuckfield RC, Baker-Austin C: Antimicrobial textiles. Handb Exp Pharmacol 2012, 211:135-152.

42. Cotton FA, Goodgame DML: Tetrakis(triphenylphosphine)-Silver(I) and (Triphenylphosphine)-Copper(I) Complexes. J Chem Soc 1960:5267-5269.

43. Schillinger U, Lücke FK: Lactic Acid Bacteria as Protective Cultures in Meat Products. Fleischwirtsch 1990, 70:1296-1299.

\section{Publish with ChemistryCentral and every scientist can read your work free of charge \\ "Open access provides opportunities to our colleagues in other parts of the globe, by allowing anyone to view the content free of charge."

$$
\text { W. Jeffery Hurst, The Hershey Company. }
$$

- available free of charge to the entire scientific community

- peer reviewed and published immediately upon acceptance

- cited in PubMed and archived on PubMed Central

- yours - you keep the copyright

Submit your manuscript here:

http://www.chemistrycentral.com/manuscript/<smiles>c1ccccc1</smiles>

Chemistry Central 